\title{
Acute Disseminated Encephalomyelitis: Current Understanding and Controversies
}

\author{
Nathan P. Young, D.O., ${ }^{1}$ Brian G. Weinshenker, M.D., ${ }^{1}$ \\ and Claudia F. Lucchinetti, M.D. ${ }^{1}$
}

Acute disseminated encephalomyelitis (ADEM) is an uncommon monophasic idiopathic inflammatory demyelinating disease. Available diagnostic criteria do not reliably distinguish it from first presentations of relapsing diseases such as multiple sclerosis (MS) and neuromyelitis optica (NMO). In this article, we review current concepts about ADEM and what distinguishes it from other idiopathic inflammatory demyelinating disease, and we highlight controversial aspects and diagnostic problems. We review pathological differences between ADEM and MS in terms of their utility in the diagnosis of ADEM. Finally, we present a practical approach for management of patients suspected of having ADEM when the diagnosis is uncertain.

KEYWORDS: Acute disseminated encephalomyelitis, postinfectious encephalomyelitis, perivenous encephalomyelitis, idiopathic inflammatory demyelination disease

\begin{abstract}
Acute disseminated encephalomyelitis (ADEM) is traditionally considered a monophasic inflammatory demyelinating disorder with pleiotropic clinical manifestations, which usually include encephalopathy, but variably include other focal or multifocal syndromes suggestive of a central nervous system (CNS) inflammatory demyelinating disorder, including optic neuritis $(\mathrm{ON})$ and myelitis. Consequently, ADEM is often considered in the differential diagnosis of a clinically isolated (demyelinating) syndrome (CIS). However, most CISs, especially in adults, are harbingers of multiple sclerosis and, therefore, of future relapses. The hallmark of ADEM is its monophasic course. Therefore, most physicians treat ADEM with short-term treatments rather than long-term disease-modifying therapies. However, early and accurate distinction between ADEM and other inflammatory demyelinating disorders, especially multiple sclerosis (MS) and neuromyelitis optica (NMO), is important for prognosis and
\end{abstract}

treatment because many patients with MS or NMO, particularly those with aggressive fulminant disease, may benefit from early disease-modifying therapy to suppress ongoing and future relapses.

Recent retrospective and prospective series suggest clinical, neuroimaging, and laboratory characteristics of ADEM that may be helpful in distinguishing ADEM from MS. ${ }^{1-14}$ Collectively, this literature suggests ADEM should be considered when one or more of the following are present: multifocal, polysymptomatic initial presentation; age younger than 10 years; signs and symptoms of meningoencephalitis; encephalopathy; bilateral ON; cerebrospinal fluid (CSF) pleocytosis without oligoclonal bands; magnetic resonance imaging (MRI)-detected lesions involving structures not typically affected in MS such as the deep gray matter or cortex; and MRI-detected lesions that are large and exhibit indistinct borders and enhancement following gadolinium administration. ${ }^{15}$
${ }^{1}$ Department of Neurology, Mayo Clinic College of Medicine, Rochester, Minnesota.

Address for correspondence and reprint requests: Claudia F. Lucchinetti, M.D., Department of Neurology, Mayo Clinic College of Medicine, 200 First Street SW, Rochester, MN 55905 (e-mail: cluccinetti@mayo.edu).
Multiple Sclerosis and the Spectrum of CNS Inflammatory Demyelinating Diseases; Guest Editor, Claudia F. Lucchinetti, M.D.

Semin Neurol 2008;28:84-94. Copyright (C) 2008 by Thieme Medical Publishers, Inc., 333 Seventh Avenue, New York, NY 10001, USA. Tel: +1(212) 584-4662.

DOI 10.1055/s-2007-1019130. ISSN 0271-8235. 
This review focuses on the clinical, laboratory, and neuroimaging characteristics, which are proposed by recent series to be helpful in distinguishing ADEM from other monophasic inflammatory demyelinating disorders. However, we emphasize that these proposed features of ADEM are mostly based on retrospective diagnoses from patients fulfilling arbitrary and nonstandardized criteria for ADEM. Therefore, the diagnostic utility of these clinical characteristics remains unclear in the absence of a prospective validation study using a clinical or clinicopathological gold standard. Because ADEM has been variably defined, some recent series have further blurred the distinction between ADEM and MS by proposing an expanded spectrum of ADEM that includes multiphasic or recurrent forms. ${ }^{1,3,10,12,16,17} \mathrm{We}$ consider pathological differences between ADEM and MS and whether they might serve as a gold standard to distinguish ADEM from other inflammatory demyelinating disorders and inform the debate on how to define ADEM.

\section{CLINICAL DEFINITIONS OF ACUTE DISSEMINATED ENCEPHALOMYELITIS}

There are no accepted, prospective, or pathologically verified clinical diagnostic criteria for ADEM. Early retrospective studies suffered from broad inclusion criteria, which almost certainly included cases of probable first presentations of $\mathrm{MS}^{2,6,8,10-12,18-20}$ and NMO., ${ }^{3,10-12}$ Building on clinicopathological and retrospective studies of ADEM, Mikaeloff et al have applied the most restricted definition of ADEM to date in a prospective study of children:

The occurrence in a previously healthy child of acute symptoms associating the following at onset: more than one neurological deficit ("polysymptomatic" onset); change in mental state; and any combination of alterations seen on MRI, providing that these included white matter lesions. ${ }^{20}$

Although the Mikaeloff et al criteria predicted a monophasic course in most patients over a mean duration of follow-up of $5.5+/-3.6$ years, $18 \%$ of patients still went on to have a relapse at a different CNS site than the first attack and appeared to have a clinical course consistent with MS. ${ }^{20}$ Mikaeloff et al suggested a diagnosis of MS in patients with recurrence, but others have concluded that such cases provide evidence that ADEM can relapse or recur, a controversy that will be discussed in more detail below. ${ }^{1,6,8,10,12,21}$ Pathological data are absent from all large series of clinically diagnosed ADEM.

The first set of consensus diagnostic criteria for ADEM were recently proposed by the International Pediatric MS Study Group (IPMSSG), a group of adult and pediatric neurologists and experts in genetics, epidemiology, neuropsychology, nursing, and immunology organized by the National Multiple Sclerosis Society. $^{22,23}$ These criteria are similar to those used by Mikaeloff et al and are outlined in Table 1. The IPMSSG does not propose these criteria as final and emphasizes the need for prospective validation over the next 10 to 20 years. These consensus criteria were developed for children ( $<10$ years). It is unclear whether any clinical diagnostic criteria for ADEM should be different in adult patients. Although these criteria are conservative by requiring encephalopathy, the most specific clinical feature that distinguishes ADEM from MS, they should be used with caution in clinical practice and have not yet been reliably proven to distinguish between either fulminant MS or other inflammatory demyelinating disorders or between ADEM and other acute leukoencephalopathies of other etiology.

\section{PATHOLOGICAL DEFINITIONS}

The pathological hallmark of ADEM is perivenular inflammation with limited "sleeves of demyelination." ${ }^{24-28}$ In some cases, larger areas of demyelination occur secondary to coalescence of many perivenous demyelinating lesions. ${ }^{26-29}$ Although perivascular inflammation is also a feature of MS pathology, the patterns of demyelination in ADEM stand in contrast to the confluent sheets of macrophage infiltration admixed with reactive astrocytes in completely demyelinated regions that are typical of an MS plaque. ${ }^{30}$ Acute hemorrhagic leukoencephalitis (AHLE) is pathologically similar to ADEM but additionally exhibits petechial hemorrhage and venular necrosis. ${ }^{24}$ The pathology of ADEM is very similar to the monophasic experimental autoimmune encephalomyelitis (EAE) animal model of acute monophasic inflammatory demyelination. ${ }^{24,25,30}$ A complete description of the pathology of ADEM and other idiopathic inflammatory demyelinating disorders is beyond the scope of this review and is reviewed elsewhere. $^{24,25,27,30,31}$

The pathological difference between perivenous and confluent demyelination suggests that brain histopathology is a possible diagnostic gold standard and may be clinically useful for distinguishing ADEM from MS and other idiopathic inflammatory demyelinating disorders. However, the true utility of brain biopsy in distinguishing ADEM from MS and other idiopathic inflammatory demyelinating disorders has not yet been examined. The clinical characteristics of a large cohort of patients with biopsy-proven confluent demyelinating disease (excluding patients with perivenous demyelination) were found to be similar to a population-based MS cohort. ${ }^{32}$ It is unknown whether a perivenous pattern of demyelination predicts a monophasic course or whether the presence of confluent demyelination predicts future 
Table 1 International Pediatric MS Study

Group-Consensus Definitions

\section{Monophasic ADEM}

- A first clinical event with a presumed inflammatory or demyelinating cause, with acute or subacute onset that affects multifocal areas of the CNS; the clinical presentation must be polysymptomatic and must include encephalopathy, which is defined as one or more of the following:

- Behavioral change occurs, e.g., confusion, excessive irritability.

- Alteration in consciousness occurs, e.g., lethargy, coma.

- Event should be followed by improvement, clinically, on MRI, or both, but there may be residual deficits.

- Patient has no history of a clinical episode with features of a prior demyelinating event.

- No other etiologies can explain the event.

- New or fluctuating symptoms, signs, or MRI findings occurring within 3 months of the inciting ADEM event are considered part of the acute event.

- Neuroimaging shows focal or multifocal lesion(s), predominantly involving white matter, without radiologic evidence of previous destructive white matter changes.

- Brain MRI, with FLAIR or T2-weighted images, reveals large $(>1$ to $2 \mathrm{~cm}$ ) lesions that are multifocal, hyperintense, and located in the supratentorial or infratentorial white matter regions; gray matter, especially basal ganglia and thalamus, is frequently involved.

- In rare cases, brain MR images show a large single lesion (1 to $2 \mathrm{~cm}$ ), predominantly affecting white matter.

- Spinal cord MRI may show confluent intramedullary lesion(s) with variable enhancement, in addition to abnormal brain MRI findings specified previously.

\section{Recurrent ADEM}

- New event of ADEM occurs with recurrence of the initial symptoms and signs 3 or more months after the first ADEM event without involvement of new clinical areas by history, examination, or neuroimaging.

- Event does not occur while on steroids and occurs at least 1 month after completing therapy.

- MRI shows no new lesions; original lesions may have enlarged

- No better explanation exists.

\section{Multiphasic ADEM}

- ADEM is followed by a new clinical event also meeting criteria for ADEM, but involving new anatomic areas of the CNS as confirmed by history, neurologic examination, and neuroimaging.

- The subsequent event must occur (1) at least 3 months after the onset of the initial ADEM event and (2) at least 1 month after completing steroid therapy.

- The subsequent event must include a polysymptomatic presentation, including encephalopathy, with neurologic symptoms or signs that differ from the initial event (mental status changes may not differ from the initial event).
Table 1 (Continued)

- The brain MRI must show new areas of involvement but also demonstrate complete or partial resolution of those lesions associated with the first ADEM event.

ADEM, acute disseminated encephalomyelitis; CNS, central nervous system; MRI, magnetic resonance imaging; FLAIR, fluid-attenuated inversion recovery.

From Krupp LB, Banwell B, Tenembaum S, for the International Pediatric MS Study Group. Consensus definitions proposed for pediatric multiple sclerosis and related disorders. Neurology 2007;68(suppl 2):S7-S12

relapse or progression. The significance of these patterns of demyelination will be clarified as expert neuropathological examination with attention to these patterns of demyelination is combined with prolonged prospective clinical and neuroimaging follow-up over 10 to 20 years, as proposed by the IPMSSG. So far, only rare case reports have clearly described the clinical and neuroimaging correlations in patients with perivenous demyelination since the last large clinicopathological series published in 1975..$^{6,10,33-37}$ Importantly, all of these cases were monophasic, most were fatal, ${ }^{6,33,35,36}$ and one case presented with a focal brainstem syndrome. ${ }^{34}$

\section{ANTECEDENT INFECTION AND VACCINATION}

Evidence of an infectious etiology associated with ADEM is supported by winter and spring seasonal peaks in presentation observed in some studies. ${ }^{8,10}$ Infection may trigger the subsequent autoimmune attack on the CNS, possibly via "molecular mimicry." 38 Numerous infections, mostly viral, and other immunological triggers, such as vaccinations, have been associated with ADEM and are listed elsewhere. ${ }^{39-41}$

Although ADEM is often associated with recent vaccination or infection, the occurrence of these events has not been included in the definition of ADEM in most studies, including the new consensus clinical criteria. $^{1-3,6,8,11,19,22,42,43}$ Only four series described patients with a clear antecedent history of infection or vaccination. ${ }^{5,9,12,14}$ The term ADEM in this article includes cases with and without a clear antecedent history of infection or vaccination. This definition is consistent with currently proposed clinical criteria, ${ }^{1,20,22}$ and assumes that there is a unique immune-mediated CNS process with a distinct pathophysiology that is independent of the immune trigger, which is often not apparent in the clinical history, even when present. Omitting the presence of a preexisting infection or vaccination from the criteria for $A D E M$ is supported by the significant proportion of likely ADEM patients evaluated prospectively, $1,20,42$ retrospectively, , $^{2,3,6,10,11,19}$ and pathologically $24,26,27,33-36,44$ who do not have a prior history of infection or vaccination. A history of antecedent infection or vaccination may increase the 
likelihood of ADEM, but also occurs with greater frequency in patients with first presentation of MS. ${ }^{45,46}$ Therefore, a strict requirement of an antecedent infection or vaccination is neither specific nor sensitive for ADEM.

\section{YOUNGER AGE AT PRESENTATION}

ADEM is more frequent in children. In one study of children with ADEM living in San Diego County, California, the incidence was estimated to be at least $0.4 / 100,000 / \mathrm{y}^{6}{ }^{6}$ The incidence of ADEM in adult $\mathrm{pa}^{-}$ tients has not been evaluated. Pediatric patients meeting Mikaeloff et al criteria for ADEM presented at a mean age of 7.1 years versus a mean of 12.0 years for MS. ${ }^{42}$ Five percent of MS patients present at an age younger than 16 years, but MS patients have been reported to present as young as 1 year of age. ${ }^{47}$ However, as the spectrum of pediatric MS has expanded, some evidence suggests that an "ADEM-like" presentation in pediatric MS may be underrecognized. ${ }^{48}$ Although patients presenting with demyelinating disease before age 10 years may be more likely to have ADEM than MS, ${ }^{15,42}$ considerable overlap in age of presentation limits the utility of using age as a discriminating factor. ${ }^{15}$

\section{SIGNS AND SYMPTOMS OF MENINGOENCEPHALITIS}

Many cases of fatal ADEM have evidence of lymphocytic meningitis on histopathologic examination. ${ }^{24,25}$ When compared with patients who are eventually diagnosed with MS, several clinical symptoms of meningoencephalitis are consistently associated with ADEM, including encephalopathy, seizures, fever, headache, and meningeal signs. ${ }^{2,10}$ These findings, however, are only present in some ADEM patients (Table 1). This presentation may be more frequent in pediatric MS exacerbations ${ }^{48}$ but are likely less common in adults. ${ }^{2}$

Encephalopathy has been emphasized as a key distinguishing characteristic of ADEM in children. ${ }^{20,22,23,42}$ This is best supported by fatal cases with pathological confirmation of perivenous demyelination characteristic of ADEM, which were documented to have early changes in mental status and decline in level of consciousness over days in all cases (albeit severe fatal cases). ${ }^{24,25,28}$ "Encephalopathy" as currently applied ${ }^{20,22}$ is not precisely defined and may be confounded by postictal state or focal demyelinating lesions that may cause aphasia, frontal behavioral syndromes, or parietal-occipital visual spatial syndromes, which may be confused with a diffuse encephalopathy. In cases of apparent ADEM with encephalopathy, the possibility of unidentified infectious encephalitis remains possible in some cases, especially from retrospective series, which did not apply a standardized broad infectious workup to each pa- tient. ${ }^{2,10,11,49}$ Most would agree that a meningoencephalitic presentation is atypical of MS and raises the clinical suspicion of ADEM. If encephalopathy is excluded, then meningoencephalitis symptoms may have merit in diagnostic criteria for ADEM, but the sensitivity may be low. The specificity of encephalopathy is likely dependent on how precisely it is defined. A change in level of arousal or consciousness may be a more specific definition that more readily discriminates ADEM from even atypical presentations of MS.

\section{BILATERAL OPTIC NEURITIS: ADEM OR NMO?}

Dale et al found that $23 \%$ of 40 patients diagnosed with ADEM presented with bilateral ON. Conversely, in the same study, unilateral $\mathrm{ON}$ was only present in patients eventually reclassified as MS, none of whom had bilateral ON. ${ }^{10}$ Although infrequently present, the occurrence of bilateral rather than unilateral $\mathrm{ON}$ was felt to potentially distinguish ADEM from MS; this robust difference was not reproduced in later studies using diagnostic criteria, which were even more restrictive (Table 2). Bilateral ON is uncommon in MS, but is a well-recognized manifestation of NMO. Cases of NMO may contaminate some ADEM series. ${ }^{1,9-12,21}$ Although early diagnostic criteria for NMO suggested that the brain MRI be relatively free of white matter lesions, recent publications provide evidence of a broader spectrum, including lesions consistent with ADEM and MS. ${ }^{50}$ Patients with NMO have antibodies to the aquaporin- 4 water channel, a recently identified novel biomarker that may be pathogenic. ${ }^{51}$ NMO-immunoglobulin ( $\mathrm{Ig}$ ) G is positive in as many as $76 \%$ of cases of clinical NMO, but is not an absolute criterion for diagnosis. ${ }^{51-53}$ Early treatment of NMO may reduce the risk of severe disabling relapses. ${ }^{52,54}$ NMO-IgG serological testing became available only recently and has not been evaluated in the ADEM series published to date. Bilateral ON is likely less specific for ADEM than originally suggested by Dale et $\mathrm{al}^{10}$ and exemplifies potential confusion of ADEM with NMO, which are likely pathophysiologically different. Future studies of ADEM should include NMO-IgG testing in all clinically possible cases ( $\mathrm{ON}$, myelitis alone, or a combination of the two).

\section{POLYSYMPTOMATIC, MULTIFOCAL INITIAL PRESENTATION}

Recent studies of ADEM only included patients with polysymptomatic presentations, excluding patients with monosymptomatic ON, transverse myelitis, brainstem encephalitis, or cerebellitis. ${ }^{1,2,6,9,10,42}$ However, some have argued that many monosymptomatic patients have a monophasic course and therefore should be considered to have limited forms of ADEM. ${ }^{11,12}$ Complicating 


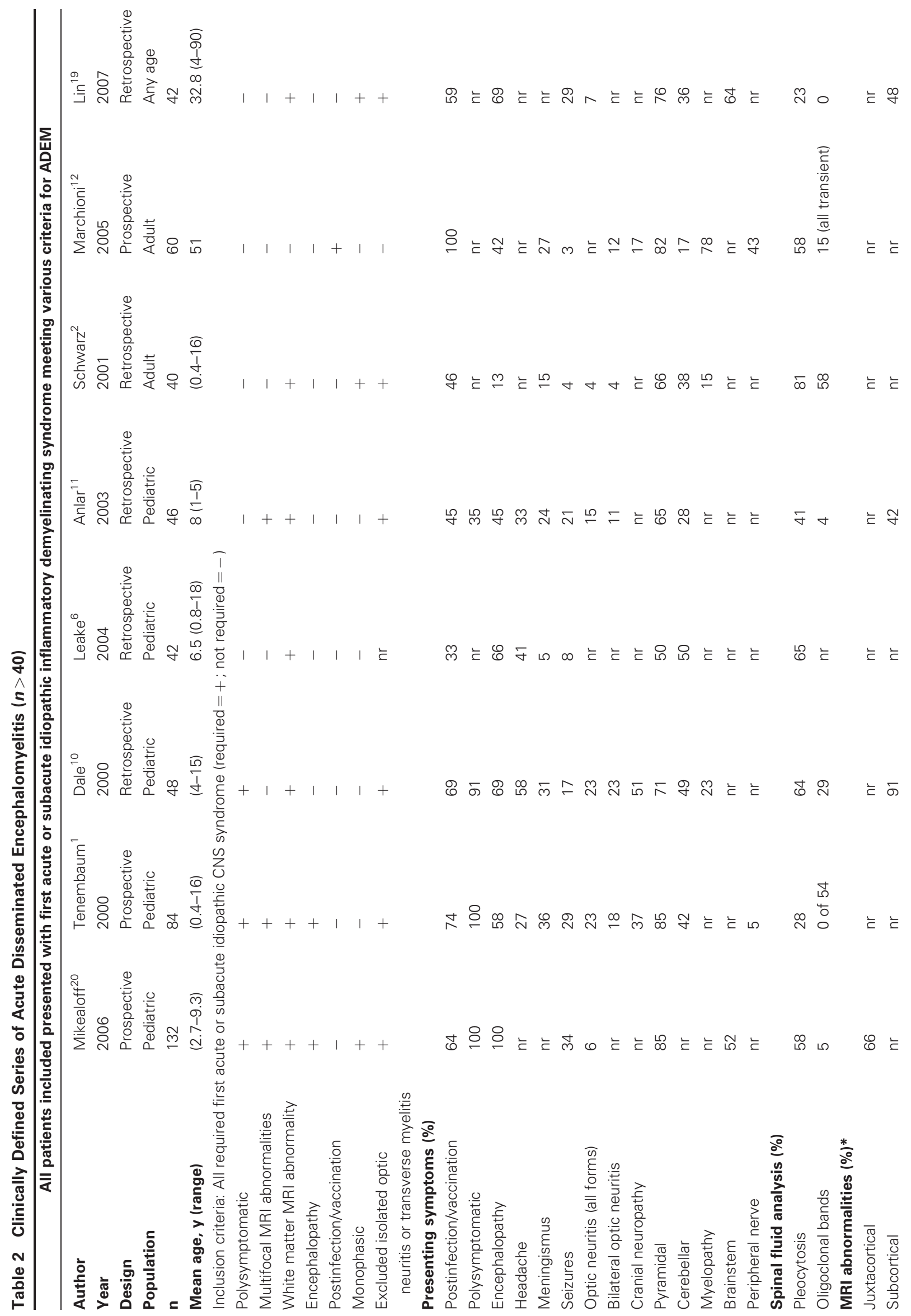




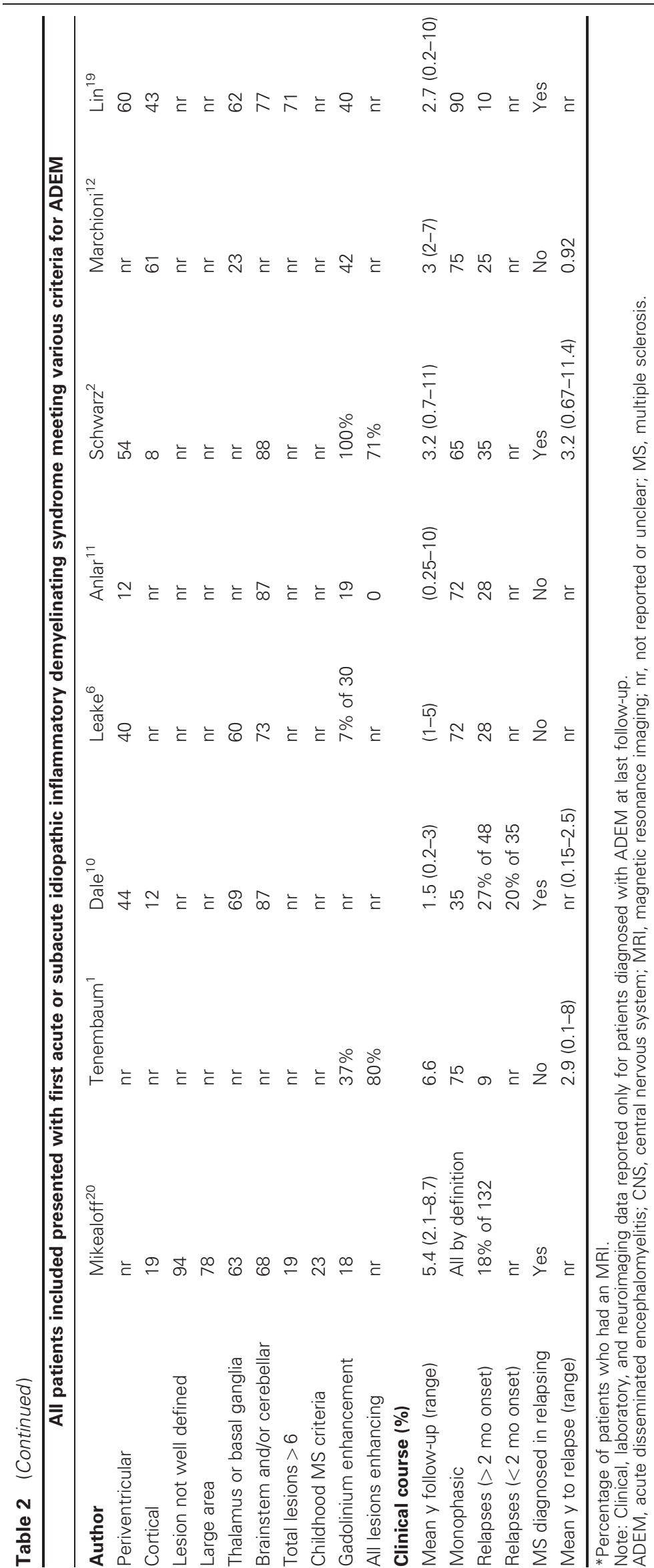


matters, patients with monosymptomatic presentations but with multifocal MRI abnormalities have a higher risk of subsequent diagnosis of MS than those who do not. In a recent retrospective study of 296 patients with a first attack of otherwise idiopathic demyelinating disease, Mikaeloff et al required a polysymptomatic presentation with mental status change, multifocal MRI abnormalities, and a monophasic course over a mean of 2.9 years for the final diagnosis of ADEM. ${ }^{42}$ A polysymptomatic presentation was not specific for ADEM, and occurred in $67 \%$ of 168 patients with MS in the same series. ADEM is pathologically diffuse and multifocal. ${ }^{24,25,28} \mathrm{~A}$ polysymptomatic presentation may be a marker of the diffuse underlying pathology of ADEM, but is nonspecific. In patients with a monosymptomatic presentation, multifocal MRI abnormalities may be surrogate markers of a multifocal process in patients with a first presentation of an idiopathic inflammatory demyelinating disorder. If the hallmark of ADEM pathology is perivenous demyelination, then brain biopsy of focal monosymptomatic lesions may help resolve the question of whether these cases should be included in the ADEM spectrum. Only two well-documented cases of perivenous demyelination limited to the brainstem are reported in the literature. ${ }^{55,56}$ We are not aware of any reports of focal supratentorial lesions with well-documented perivenous pathology. On the contrary, most cases of focal supratentorial demyelination reveal confluent demyelination, which is pathologically more consistent with MS pathology, although some have argued these may be intermediate lesions along an ADEM-to-MS spectrum. ${ }^{57}$

\section{ASSOCIATED POLYRADICULOPATHY}

A recent series of adult patients presenting with a CNS syndrome after infection or vaccination were prospectively followed over a minimum of 2 years; $43 \%$ of the 75 patients included were found to have peripheral nervous system (PNS) involvement, which was usually demyelinating and subclinical. This finding was likely due to the use of broad inclusion criteria (Table 2) and because all patients underwent extensive prospective neurophysiologic testing. ADEM was only rarely reported to be associated with PNS involvement before this study. ${ }^{58-61}$ Notably, the diagnosis was based on less specific criteria than other recent studies of ADEM. Also, autopsy series ${ }^{24,26,27}$ and the clinically defined series of ADEM do not report evidence of PNS pathology. Further study is needed to determine if PNS involvement is part of the ADEM spectrum.

\section{SPINAL FLUID PLEOCYTOSIS WITHOUT OLIGOCLONAL BANDS}

Before diagnosing ADEM, infection must be excluded by CSF analysis and culture. The CSF may be normal in
ADEM or reveal a lymphocytic pleocytosis (Table 2), in contrast to cases of MS, which rarely have a pleocytosis. Detection of oligoclonal bands (OCBs) may be helpful in predicting a subsequent diagnosis of MS, but the true utility is unknown because as many as $58 \%$ of adult ${ }^{2}$ and $29 \%$ of pediatric ${ }^{10}$ cases with ADEM have OCBs. Anecdotally, the bands should resolve in ADEM but are more likely to persist in MS., ${ }^{1,47}$ This was true in a series of nine ADEM patients who initially had OCBs, which resolved when analysis was repeated 6 days to 6 months later. ${ }^{12}$ The presence of OCBs on initial presentation is not specific for MS; however, if OCBs persist, then a diagnosis of MS is more likely. ${ }^{11}$

\section{MAGNETIC RESONANCE IMAGING}

Although MRI neuroimaging is useful for the diagnosis of ADEM and exclusion of other diagnoses, the consensus ADEM criteria emphasize clinical criteria and underplay the role of MRI to establish a diagnosis. In clinically defined cases of ADEM, the MRI will often demonstrate multifocal areas of increased T2-weighted (T2W) signal abnormalities in the CNS white matter, with or without gray matter involvement. Some authors have proposed that ADEM lesions are indistinct and lack sharply defined borders characteristic of MS lesions. ${ }^{4,10}$ Although ADEM lesions (of similar age) should all hypothetically enhance with gadolinium, this finding is rarely seen, and gadolinium enhancement may even be absent. 2,4,8,10,12,62,63

Early MRI series identified overlap in lesion location and distribution between ADEM and MS, but also highlighted features of ADEM that are unusual in MS, such as symmetric bilateral disease, relative sparing of the periventricular white matter, or deep gray matter involvement. ${ }^{64}$ Absolute and relative periventricular sparing on MRI is typical of ADEM, and was present in $78 \%$ of patients with ADEM reported by Dale et al. However, 22\% of ADEM patients had a periventricular lesion pattern indistinguishable from that seen in $\mathrm{MS}^{10}$

Mikaeloff et al prospectively found that corpus callosum long axis lesions (Dawson's fingers), together with the finding of only well-defined lesions, were completely specific predictors of relapse and, accordingly, of MS over a mean of 4.9 years. However, only $21 \%$ of patients presenting with a first episode of demyelination had this finding. This study highlights the difficulty of using the initial MRI in identifying patients with increased risk of relapsing disease (MS), or those with a truly monophasic process. ${ }^{4}$ In contrast with other studies, lesions of the thalamus and basal ganglia were not significantly different between ADEM or MS patients in this study. 
Even when the MRI seems typical of either ADEM or MS, the findings remain relatively nonspecific, and a broad differential diagnosis of potential mimics of idiopathic inflammatory demyelinating disorders must be carefully considered. ${ }^{65}$ MRI criteria may be most helpful in combination with clinical criteria in verifying a multifocal demyelinating process or suggesting an alternative pathology. Although the possibility of MRI-negative ADEM has been proposed, ${ }^{5,9,10,66}$ it remains unclear whether an unidentified cause of encephalopathy other than ADEM may have been present in such cases. The recently published consensus criteria require an abnormal MRI and require that lesions should be larger than 1 to $2 \mathrm{~cm}$ to be compatible with ADEM. The current consensus criteria for ADEM suggest that rarely a large focal lesion may be compatible with $\mathrm{ADEM},{ }^{22}$ even though some may regard this as a tumefactive presentation of MS. This question should be easily resolved by clearly documenting the pathology (perivenous or confluent demyelinating) in cases in which brain biopsy may have been performed to exclude neoplasm, and correlating the pattern of demyelination with clinical course.

\section{ADVANCED NEUROIMAGING TECHNIQUES}

Advanced neuroimaging techniques, such as diffusion tensor imaging (DTI) and magnetic transfer imaging (MTI), may provide a better assessment of the underlying histopathology than an increase in T2W signal on conventional MRI. A small study using DTI found a significant difference in basal ganglia DTI signal between ADEM and MS, even when there was no difference in basal ganglia T2W signal between groups. ${ }^{67}$ Magnetization transfer and diffusion tensor MR imaging may also be helpful in identifying involvement of the so-called "normal-appearing white matter," which is often abnormal in MS but may not be in ADEM. ${ }^{68}$ Quantitative MRI techniques have also suggested that progressive ventricular enlargement and gray matter atrophy are potential markers for MS in patients presenting with clinically isolated syndromes. ${ }^{69,70} \mathrm{Ad}-$ vanced neuroimaging techniques should be studied further as this is a promising avenue for noninvasively approximating the underlying tissue pathology.

\section{MULTIPHASIC AND RECURRENT ADEM}

The monophasic clinical hallmark of ADEM is challenged by cases of recurrent ADEM, reported mainly in isolated case reports without pathological confirmation. A single case series of presumed recurrent ADEM at the previously affected brain site with accompanying pathology has been reported. ${ }^{16}$ This series has been cited as evidence of the existence of recurrent ADEM. However, the description of pathology in this series was limited to "diffuse demyelination" and one published photomicrograph demonstrating confluent demyelination was consistent with classic MS pathology. This diagnosis of recurrent ADEM was based on clinical presentation rather than characteristic perivenular ADEM pathology. The lack of pathological evidence is a common limitation of many clinicopathologic series of ADEM.

Even in the two large prospective studies of children with the most restrictive clinical criteria for ADEM, between $10 \%$ and $18 \%$ of patients subsequently had relapses. ${ }^{1,20}$ The Tenembaum et al cohort ${ }^{1}$ was systematically evaluated to rule out infection or other causes of a similar syndrome. Serial neuroimaging was performed at presentation, and follow-up examinations were performed over a mean of 6.6 years. This study identified 76 monophasic patients with a clinically and radiographically monophasic course, implying a separate pathophysiology than typical cases of MS. However, $10 \%$ of their 84 patients had one relapse at a previously unaffected site with a mean inter-attack interval of 2.9 years (range 2 months to 8 years) and subsequently remained relapse free for a mean of 8.2 years. After the relapse, these patients did not develop new subclinical lesions on yearly prospective repeat MRI scans. All were OCB negative. None of those with recurrences had biopsy verification of ADEM pathology. Based on these observations, the authors proposed a "biphasic" form of ADEM. ${ }^{1}$ A similar group of relapsing patients are described in the Mikaeloff cohort ${ }^{20}$ (18\% of $132 \mathrm{pa}^{-}$ tients); however, Mikaeloff et al diagnosed MS rather than ADEM in this circumstance. Interestingly, in the Tenembaum cohort, brain biopsy was reported from four patients with a monophasic course, the findings of which were more typical of confluent MS pathology.

The recent consensus criteria for ADEM attempt to clarify the terminology used to describe cases of possible ADEM that are not monophasic. A case is "recurrent" if a subsequent attack is stereotypical of the first attack and there is no evidence of involvement of a different part of the CNS clinically or by MRI. A case is "multiphasic" if evidence is found of new symptoms or of involvement of a different part of the CNS than that of the initial attack (Table 1). The terms "relapsing" and "biphasic" ADEM have been appropriately discarded by the consensus group. ${ }^{22}$

\section{MONOPHASIC UNTIL PROVED OTHERWISE}

We propose that ADEM be defined as a strictly monophasic disease until more definitive data prove otherwise. The duration of follow-up required to verify a monophasic course is uncertain. Follow-up as long as 10 years may be needed, as the longest duration until first relapse was 8 years in the Tenembaum study ${ }^{1,10}$ and a mean of $5.4+/-3.3$ years in the Mikaeloff study. ${ }^{20}$ Attacks 
occurring within 3 months of an initial attack or during a steroid taper are not considered new attacks based on the consensus clinical criteria. The long-term prospective follow-up of patients diagnosed with ADEM by the IPMSSG will provide important natural history data on ADEM and its potential relationship with MS.

\section{RECOMMENDATIONS FOR CLINICIANS}

Although there is progress in defining ADEM, reliable clinical diagnostic criteria are still not established. Even the most restrictive criteria for ADEM do not reliably predict a monophasic course in patients presenting with first attacks of an idiopathic inflammatory demyelinating disorder. Recognizing the clinical and neuroimaging characteristics of encephalopathy-polysymptomatic presentation; MRI lesions that are large, multifocal, and involving deep gray matter and cortex; CSF pleocytosis without $\mathrm{OCBs}$ - also requires that other mimickers of inflammatory demyelinating disease and also first presentations of MS be considered in addition to ADEM. NMO should also be considered in patients with prominent myelitis or $\mathrm{ON}$, particularly if they are NMO-IgG positive. Once an infectious etiology has been reasonably excluded, intravenous methylprednisolone, $30 \mathrm{mg} / \mathrm{kg} / \mathrm{d}$ in children and doses up to $1000 \mathrm{mg}$ daily in adults for 3 to 5 days, is the most common treatment approach in practice, based on anecdotal evidence from case reports and clinical series. ${ }^{71}$ Intravenous immunoglobulin (IVIg) may be an effective alternative. $^{72,73} \mathrm{~A}$ brain biopsy is indicated in some patients when the diagnosis is uncertain and clinical or imaging features suggest alternative diagnoses (e.g., neoplasm). Interpretation of brain biopsy by an expert neuropathologist is essential. Clinicians should avoid the potential pitfall of diagnosing ADEM simply because there are atypical clinical features of $\mathrm{MS}$, and therefore assume a benign monophasic course. Close follow-up with serial examination and MRI within the first 3 months is recommended, as new subclinical lesions may develop on MRI and suggest the diagnosis of MS. A yearly clinical follow-up, often with repeat neuroimaging to look for evidence of new subclinical lesions, is a possible surveillance strategy for patients with an unclear idiopathic inflammatory demyelinating disorder clinical phenotype in whom initiation of immunomodulatory therapy may eventually be indicated.

\section{FUTURE DIRECTIONS}

Multiple clinical factors should be considered in combination before arriving at a probable diagnosis of ADEM including: (1) presenting signs and symptoms, (2) conventional and advanced MRI abnormalities, (3) sufficient follow-up (perhaps as long as 10 years) confirming a monophasic course based on clinical and/or neuro- imaging criteria, (4) lack of alternative diagnosis, and (5) brain histopathology when available. The IPMSSG has outlined a plan to prospectively study specific clinical diagnostic criteria in children. ${ }^{22}$ Although these criteria will likely evolve with time, an agreement on terminology and suggestive clinical criteria is an important step forward. It is unclear whether ADEM presents differently in adults than children (as presentations of MS may be different), because specific criteria such as those proposed by Mikaeloff and the IPMSSG have not been tested in adult populations. Although brain biopsy is uncommonly performed in most clinical practices, well-described clinicopathological cases distinguishing between limited perivenous and confluent demyelination may help support pathology as a gold standard, verify cases, and refine future clinical diagnostic criteria for ADEM.

\section{REFERENCES}

1. Tenembaum S, Chamoles N, Fejerman N. Acute disseminated encephalomyelitis: a long-term follow-up study of 84 pediatric patients. Neurology 2002;59(8):1224-1231

2. Schwarz S, Mohr A, Knauth M, Wildemann B, StorchHagenlocher B. Acute disseminated encephalomyelitis: a follow-up study of 40 adult patients. Neurology 2001;56(10): 1313-1318

3. Murthy SN, Faden HS, Cohen ME, Bakshi R. Acute disseminated encephalomyelitis in children. Pediatrics 2002; 110(2 Pt 1):e21

4. Mikaeloff Y, Adamsbaum C, Husson B, et al. MRI prognostic factors for relapse after acute CNS inflammatory demyelination in childhood. Brain 2004;127(Pt 9):1942-1947

5. Idrissova ZhR, Boldyreva MN, Dekonenko EP, et al. Acute disseminated encephalomyelitis in children: clinical features and HLA-DR linkage. Eur J Neurol 2003;10(5):537-546

6. Leake JA, Albani S, Kao AS, et al. Acute disseminated encephalomyelitis in childhood: epidemiologic, clinical and laboratory features. Pediatr Infect Dis J 2004;23(8):756-764

7. Rust RS. Multiple sclerosis, acute disseminated encephalomyelitis, and related conditions. Semin Pediatr Neurol 2000;7(2):66-90

8. Hynson JL, Kornberg AJ, Coleman LT, Shield L, Harvey AS, Kean MJ. Clinical and neuroradiologic features of acute disseminated encephalomyelitis in children. Neurology 2001; 56(10):1308-1312

9. Hollinger P, Sturzenegger M, Mathis J, Schroth G, Hess CW. Acute disseminated encephalomyelitis in adults: a reappraisal of clinical, CSF, EEG, and MRI findings. J Neurol 2002;249(3):320-329

10. Dale RC, de Sousa C, Chong WK, Cox TC, Harding B, Neville BG. Acute disseminated encephalomyelitis, multiphasic disseminated encephalomyelitis and multiple sclerosis in children. Brain 2000;123(Pt 12):2407-2422

11. Anlar B, Basaran C, Kose G, et al. Acute disseminated encephalomyelitis in children: outcome and prognosis. Neuropediatrics 2003;34(4):194-199

12. Marchioni E, Ravaglia S, Piccolo G, et al. Postinfectious inflammatory disorders: subgroups based on prospective follow-up. Neurology 2005;65(7):1057-1065

13. Papais-Alvarenga RM, Miranda-Santos CM, PuccioniSohler M, et al. Optic neuromyelitis syndrome in Brazilian 
patients. J Neurol Neurosurg Psychiatry 2002;73(4):429_ 435

14. Hung KL, Liao HT, Tsai ML. The spectrum of postinfectious encephalomyelitis. Brain Dev 2001;23(1):42-45

15. Dale RC, Branson JA. Acute disseminated encephalomyelitis or multiple sclerosis: can the initial presentation help in establishing a correct diagnosis? Arch Dis Child 2005; 90(6):636-639

16. Cohen O, Steiner-Birmanns B, Biran I, Abramsky O, Honigman S, Steiner I. Recurrence of acute disseminated encephalomyelitis at the previously affected brain site. Arch Neurol 2001;58(5):797-801

17. Brinar VV, Poser CM. The spectrum of disseminated encephalomyelitis. Clin Neurol Neurosurg 2006;108(3): 295-310

18. Apak RA, Kose G, Anlar B, Turanli G, Topaloglu H, Ozdirim E. Acute disseminated encephalomyelitis in childhood: report of 10 cases. J Child Neurol 1999;14(3):198201

19. Lin CH, Jeng JS, Hsieh ST, Yip PK, Wu RM. Acute disseminated encephalomyelitis: a follow-up study in Taiwan. J Neurol Neurosurg Psychiatry 2007;78(2):162-167

20. Mikaeloff Y, Caridade G, Husson B, Suissa S, Tardieu M. Acute disseminated encephalomyelitis cohort study: prognostic factors for relapse. Eur J Paediatr Neurol 2007; 11(2):90-95

21. Murthy JM, Yangala R, Meena AK, Reddy JJ. Clinical, electrophysiological and magnetic resonance imaging study of acute disseminated encephalomyelitis. J Assoc Physicians India 1999;47(3):280-283

22. Krupp LB, Banwell B, Tenembaum Sfor the International Pediatric MS Study Group. Consensus definitions proposed for pediatric multiple sclerosis and related disorders. Neurology 2007;68(suppl 2):S7-S12

23. Tenembaum S, Chitnis T, Ness J, Hahn JSfor the International Pediatric MS Study Group. Acute disseminated encephalomyelitis. Neurology 2007;68(suppl 2):S23-S36

24. Hart MN, Earle KM. Haemorrhagic and perivenous encephalitis: a clinical-pathological review of 38 cases. J Neurol Neurosurg Psychiatry 1975;38(6):585-591

25. Van Bogaert L. Post-infectious encephalomyelitis and multiple sclerosis; the significance of perivenous encephalomyelitis. J Neuropathol Exp Neurol 1950;9(3):219-249

26. Oppenheimer DR. Demyelinating diseases. In: Blackwood W, Corsellis JAN, eds. Greenfield's Neuropathology. 3rd ed. London: Edward Arnold Ltd; 1976:470-499

27. Greenfield JG, Norman RM. Demyelinating diseases. In: Blackwood W, McMenemey WH, Meyer M, Norman RM, Russell DS, eds. Greenfield's Neuropathology. 2nd ed. London: Arnold; 1971

28. Turnbull HM, McIntosh J. Encephalomyelitis following vaccination. Br J Exp Pathol 1926;7:181-222

29. Malamud N. Sequelae of postmeasles encephalomyelitis: a clinicopathologic study. Arch Neurol Psychiatry 1939; 41:943-954

30. Lucchinetti CF, Parisi J, Bruck W. The pathology of multiple sclerosis. Neurol Clin 2005;23(1):77-105vi

31. Greenfield JG. The pathology of measles encephalomyelitis. Brain 1929;52:171-195

32. Pittock SJ, McClelland RL, Achenbach SJ, et al. Clinical course, pathological correlations, and outcome of biopsy proved inflammatory demyelinating disease. J Neurol Neurosurg Psychiatry 2005;76(12):1693-1697
33. Shintaku M, Matsumoto R. Disseminated perivenous necrotizing encephalomyelitis in systemic lupus erythematosus: report of an autopsy case. Acta Neuropathol 1998;95(3): 313-317

34. Miller DH, Scaravilli F, Thomas DC, Harvey P, Hirsch NP. Acute disseminated encephalomyelitis presenting as a solitary brainstem mass. J Neurol Neurosurg Psychiatry 1993;56(8): 920-922

35. Case records of the Massachusetts General Hospital. Weekly clinicopathological exercises. Case 37-1995. A 6-year-old boy with a rash, meningismus, and diplegia. N Engl J Med 1995; 333(22):1485-1493

36. Koch M, den Dunnen W, Sie OG, De Keyser J. A fatal demyelinating illness in a young woman 10 weeks post partum. Lancet Neurol 2005;4(2):129-134

37. Silver B, McAvoy K, Mikesell S, Smith TW. Fulminating encephalopathy with perivenular demyelination and vacuolar myelopathy as the initial presentation of human immunodeficiency virus infection. Arch Neurol 1997;54(5):647650

38. Fujinami RS, Zurbriggen A. Is Theiler's murine encephalomyelitis virus infection of mice an autoimmune disease? APMIS 1989;97(1):1-8

39. Wingerchuk DM. Postinfectious encephalomyelitis. Curr Neurol Neurosci Rep 2003;3(3):256-264

40. Mihai C, Jubelt B. Post-infectious encephalomyelitis. Curr Neurol Neurosci Rep 2005;5(6):440-445

41. Menge T, Hemmer B, Nessler S, et al. Acute disseminated encephalomyelitis: an update. Arch Neurol 2005;62(11): 1673-1680

42. Mikaeloff Y, Suissa S, Vallee L, et al. First episode of acute CNS inflammatory demyelination in childhood: prognostic factors for multiple sclerosis and disability. J Pediatr 2004; 144(2):246-252

43. Mikaeloff Y, Caridade G, Assi S, Suissa S, Tardieu M. Prognostic factors for early severity in a childhood multiple sclerosis cohort. Pediatrics 2006;118(3):1133-1139

44. Prineas JW, McDonald WI, Franklin RJM. Demyelinating diseases. In: Graham D, Lantos P, eds. Greenfield's Neuropathology. Vol. 2, 7th ed. New York: Oxford University Press; 2002

45. Marrie RA, Wolfson C, Sturkenboom MC, et al. Multiple sclerosis and antecedent infections: a case-control study. Neurology 2000;54(12):2307-2310

46. Sibley WA, Bamford CR, Clark K. Clinical viral infections and multiple sclerosis. Lancet 1985;1(8441):1313-1315

47. Banwell BL. Pediatric multiple sclerosis. Curr Neurol Neurosci Rep 2004;4(3):245-252

48. Ruggieri M, Iannetti P, Polizzi A, Pavone L, Grimaldi LM. Multiple sclerosis in children under 10 years of age. Neurol Sci 2004;25(S4):S326-S335

49. Gupte G, Stonehouse M, Wassmer E, Coad NA, Whitehouse WP. Acute disseminated encephalomyelitis: a review of 18 cases in childhood. J Paediatr Child Health 2003;39(5):336342

50. Pittock SJ, Lennon VA, Krecke K, Wingerchuk DM, Lucchinetti CF, Weinshenker BG. Brain abnormalities in neuromyelitis optica. Arch Neurol 2006;63(3):390-396

51. Lennon VA, Wingerchuk DM, Kryzer TJ, et al. A serum autoantibody marker of neuromyelitis optica: distinction from multiple sclerosis. Lancet 2004;364(9451):2106-2112

52. Wingerchuk DM. Diagnosis and treatment of neuromyelitis optica. Neurologist 2007;13(1):2-11 
53. Wingerchuk DM. Acute disseminated encephalomyelitis: distinction from multiple sclerosis and treatment issues. Adv Neurol 2006;98:303-318

54. Weinshenker BG, Wingerchuk DM, Vukusic S, et al. Neuromyelitis optica IgG predicts relapse after longitudinally extensive transverse myelitis. Ann Neurol 2006;59(3):566569

55. Miller AA, Ramsden F. Acute necrotic myelitis and perivenous encephalomyelitis associated with hypertension and renal infection. J Clin Pathol 1967;20(6):821-825

56. Hoffman HL, Norman RM. Acute necrotic myelopathy associated with perivenous encephalomyelitis. J Neurol Neurosurg Psychiatry 1964;27:116-124

57. Kepes JJ. Large focal tumor-like demyelinating lesions of the brain: intermediate entity between multiple sclerosis and acute disseminated encephalomyelitis? A study of 31 patients. Ann Neurol 1993;33(1):18-27

58. Aimoto Y, Moriwaka F, Matsumoto A, Tashiro K, Abe K. A case of acute disseminated encephalomyelitis (ADEM) associated with demyelinating peripheral neuropathy. No To Shinkei 1996;48(9):857-860

59. Amit R, Shapira Y, Blank A, Aker M. Acute, severe, central and peripheral nervous system combined demyelination. Pediatr Neurol 1986;2(1):47-50

60. Amit R, Glick B, Itzchak Y, Dgani Y, Meyeir S. Acute severe combined demyelination. Childs Nerv Syst 1992;8(6):354356

61. Kinoshita A, Kaseda S, Yagi K, Oda M, Tanabe H. A case of acute disseminated encephalomyelitis with pathologicallyproven acute demyelinating lesion in the peripheral nervous system. Rinsho Shinkeigaku 1994;34(9):892-897

62. Caldemeyer KS, Harris TM, Smith RR, Edwards MK. Gadolinium enhancement in acute disseminated encephalomyelitis. J Comput Assist Tomogr 1991;15(4):673675

63. Caldemeyer KS, Smith RR, Harris TM, Edwards MK. MRI in acute disseminated encephalomyelitis. Neuroradiology 1994;36(3):216-220
64. Kesselring J, Miller DH, Robb SA, et al. Acute disseminated encephalomyelitis. MRI findings and the distinction from multiple sclerosis. Brain 1990;113(Pt 2):291-302

65. Bastianello S, Pichiecchio A, Spadaro M, et al. Atypical multiple sclerosis: MRI findings and differential diagnosis. Neurol Sci 2004;25(S4):S356-S360

66. Murray BJ, Apetauerova D, Scammell TE. Severe acute disseminated encephalomyelitis with normal MRI at presentation. Neurology 2000;55(8):1237-1238

67. Holtmannspotter M, Inglese M, Rovaris M, Rocca MA, Codella M, Filippi M. A diffusion tensor MRI study of basal ganglia from patients with ADEM. J Neurol Sci 2003; 206(1):27-30

68. Inglese M, Salvi F, Iannucci G, Mancardi GL, Mascalchi M, Filippi M. Magnetization transfer and diffusion tensor MR imaging of acute disseminated encephalomyelitis. AJNR Am J Neuroradiol 2002;23(2):267-272

69. Dalton CM, Brex PA, Jenkins R, et al. Progressive ventricular enlargement in patients with clinically isolated syndromes is associated with the early development of multiple sclerosis. J Neurol Neurosurg Psychiatry 2002;73(2):141-147

70. Dalton CM, Chard DT, Davies GR, et al. Early development of multiple sclerosis is associated with progressive grey matter atrophy in patients presenting with clinically isolated syndromes. Brain 2004;127(Pt 5):1101-1107

71. Keegan M, Pineda AA, McClelland RL, Darby CH, Rodriguez M, Weinshenker BG. Plasma exchange for severe attacks of CNS demyelination: predictors of response. Neurology 2002;58(1):143-146

72. Marchioni E, Marinou-Aktipi K, Uggetti C, et al. Effectiveness of intravenous immunoglobulin treatment in adult patients with steroid-resistant monophasic or recurrent acute disseminated encephalomyelitis. J Neurol 2002;249(1):100104

73. Sahlas DJ, Miller SP, Guerin M, Veilleux M, Francis G. Treatment of acute disseminated encephalomyelitis with intravenous immunoglobulin. Neurology 2000;54(6):13701372 